\title{
TRANSMISIÓN DE SEÑALES FISIOLÓGICAS PARA APLICACIONES DE TELESALUD SOBRE REDES MÓVILES, FPGA Y WEBSOCKETS
}

\section{TRANSMISSION OF PHISIOLOGICAL SIGNALS FOR TELEHEALTH APPLICATIONS ON MOBILE NETWORKS, FPGA AND WEBSOCKETS}

\author{
Ing. Jose Pablo Pinilla, Ing. Oscar Alberto Mantilla. \\ Ing. Leonardo Andrés Rodríguez, Ing. Sherneyko Plata Rangel. \\ Fundación Cardiovascular de Colombia, Grupo de Bioingeniería FCV. \\ Carrera 5a, No. 6-33, Floridablanca, Santander, Colombia. Tel: +(577) 6796470. \\ E-mail: \{josepinilla, oscarmantilla, leonardorodriguez, sherneykoplata\}@fcv.org
}

\begin{abstract}
Resumen: El presente artículo hace una revisión de las tecnologías implementadas y la caracterización de un prototipo electrónico desarrollado en la Fundacion Cardiovascular de Colombia (FCV), para monitorización remota mediante el envío de datos de parámetros fisiológicos a través de redes celulares y visualización Web a través de internet. Tecnologías como la decodificación de tramas de datos con FPGAs (Field Programmable Gate Arrays), la utilización de redes celulares GSM, WCDMA, y el empaquetamiento por websocket.
\end{abstract}

Palabras clave: Dispositivo médico, FPGA, telesalud, red móvil, websocket.

\begin{abstract}
This paper reviews the implemented technologies and characterization of an electronic prototype developed by the FCV (Fundación Cardiovascular de Colombia) for remote monitoring with the transmission of physiological parameters through a mobile network and Web visualization through internet. Technologies such as data frames decoding using FPGA, the use of GSM and WCDMA mobile networks, and frame packaging with the websocket protocol.
\end{abstract}

Keywords: Medical device, FPGA, telehealth, mobile network, websocket.

\section{INTRODUCCIÓN}

Los sistemas de telesalud constan de dos componentes principales que conectan dos ubicaciones geográficas con el fin de intercambiar información de tipo médico. Un componente es la unidad de telesalud y el otro la unidad base. Esta topología permite distintas distribuciones dependiendo del servicio a implementar, por ejemplo, el uso de la unidad de telesalud en el lugar de emergencia en conexión con la unidad base para consulta con el personal médico especializado, o tele-monitorización en casa, con ayuda opcional de personal asistencial [1].
Existen grandes redes de telesalud en el mundo que prestan los servicios de telemedicina, cuidado ubicuo, y monitorización de pacientes en casa o Home Care, entre las cuales se encuentran la OTN (Ontario Telemedicine Network) y la CTN (California Telehealth Network) de Canadá y EE.UU respectivamente. La OTN tiene proyectada la vinculación de 30.000 pacientes a sus servicios de telesalud para el 2015 a través de los programas de Teledermatology, Telestroke, Teletrauma, Telehomecare, entre otros. Para lo cual hacen uso de equipos especializados para ser utilizados por los pacientes con o sin ayuda de personal de enfermería [2]. 
Este artículo hace un recorrido por la metodología y componentes de un sistema de telesalud, y muestra los resultados de la implementación para lograr la comunicación de señales de parámetros fisiológicos mediante redes celulares. Este sistema hace parte de un Monitor de Signos Vitales (MSV) en desarrollo por el grupo de Bioingeniería de la Fundación Cardiovascular de Colombia, para uso en servicios de Telesalud para cuidado en casa o en unidades de transporte.

\section{ESTADO DEL ARTE}

Desde sus inicios, la atencion domiciliaria (Homecare) de pacientes ha probado su efectividad permitiendo un diagnostico $y$ tratamiento a pacientes recurrentes o en recuperacion mediante atencion temprana evitando asi complicaciones. La evolucion de las redes de telecomunicaciones ha permitido manejar progresivamente mayores volumenes de datos, con una mayor cobertura diversificando asi sus posibles aplicaciones.

Equipos con capacidades telemedicas se han propuesto conceptualmente desde los años 20. La implementacion de nuevas tecnologias basadas en telesalud ha permitido descentralizar este procedimiento apoyandose en la conectividad que las redes moviles proveen, facilitando accesibilidad a pacientes en zonas remotas y evitando el traslado innecesario de personal medico especializado. Actualmente existen plataformas de telesalud robustas en funcionamiento alrededor del mundo (Philips, Motiva, Cisco HealthPresence, VirtuMedix), sin embargo estas soluciones poco son utilizadas regionalmente debido a que su coste en infraestructura y plataformas sigue siendo representativo, usualmente no poseen ubicacion por GPS (de gran utilidad en movilidad de pacientes), y sus opciones de conectividad no se ajustan apropiadamente a los recursos disponibles en la geografia nacional.

En Colombia se han adelantado proyectos importantes en cuanto al diseño de dispositivos médicos que usan las Tecnologías de la Información y Telecomunicaciones (TICs). El grupo de investigación Bioingenium creó en el 2008 un dispositivo de telemonitorización de signos vitales [3], y la Fundación Cardiovascular de Colombia (FCV) creó el Centro Nacional de Telemedicina (CNT) en el año 2007, prestando servicios de Teleconsultas (5998 consultas) de diferentes especialidades, Telediagnóstico (con 22672 lecturas de paraclínicos como radiografías y
Electrocardiogramas) y TeleUCI (2.918 pacientes), para una presencia total en 23 departamentos del país que han contado con los servicios de telemedicina brindados por la FCV durante los últimos años.

Estos programas se han llevado a cabo por medio de equipos electrónicos basados en equipos de computo convencionales, desarrollados por el equipo de Bioingeniería de la FCV con capacidad de enviar la información a una unidad base en donde se decodifica y se muestra al médico especialista. La aplicación de estas herramientas han ayudado a racionalizar las remisiones a segundo nivel de una manera más segura, además de disminuir la mortalidad en pacientes de UCI [4]. El desarrollo de un nuevo equipo basado en sistemas embebidos y la actualización de la unidad base obedecen al objetivo de dar continuidad a estos servicios, al mejoramiento del mismo y la evolución de la plataforma completa de telemedicina.

\section{COMPONENTES Y METODOLOGÍA}

El monitor cuenta con un dispositivo FPGA (Field Programmable Gate-Array) con módulos en descripción de hardware en Verilog dedicados a la aceleración de tareas y un procesador Nios II [5] donde se implementa el Software de control de periféricos y empaquetamiento de datos para la comunicación, haciendo uso de un RTOS (Real Time Operating System). La transmisión de datos se realiza sobre el protocolo Websocket mediante un módulo de conexión inalámbrica con bandas WCDMA y GSM disponibles en Colombia, $850 / 1900 / 2100$ y 850/900/1800/1900 MHz respectivamente, permitiendo así el alcance de servicios de telesalud en áreas remotas con cobertura de tecnología $2 \mathrm{G}$ y superiores[6].

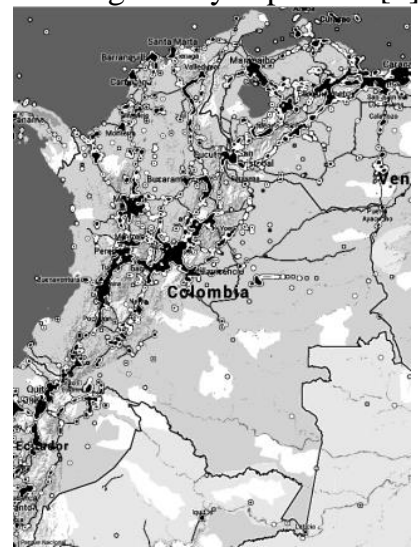

Fig. 1. Cobertura 2G/3G/4G por Movistar/Tigo/Comcel en Colombia. 


\subsection{Conexión de Datos}

Las funcionalidades proporcionadas por este sistema de comunicación permiten la prestación del servicio de telesalud en unidades de transporte hospitalario, hogares sin conexión de banda ancha, y centros de atención en regiones remotas. Estas necesidades están enmarcadas dentro del plan de tecnología Vive Digital del Gobierno de Colombia[7] con el objetivo de implementar sistemas de telesalud en 260 poblaciones rurales del país. Esta iniciativa está apoyada en el incremento y mejoramiento del uso del espectro electromagnético y con esto la cuadruplicación del número conjunto de usuarios de internet móvil y fijo.

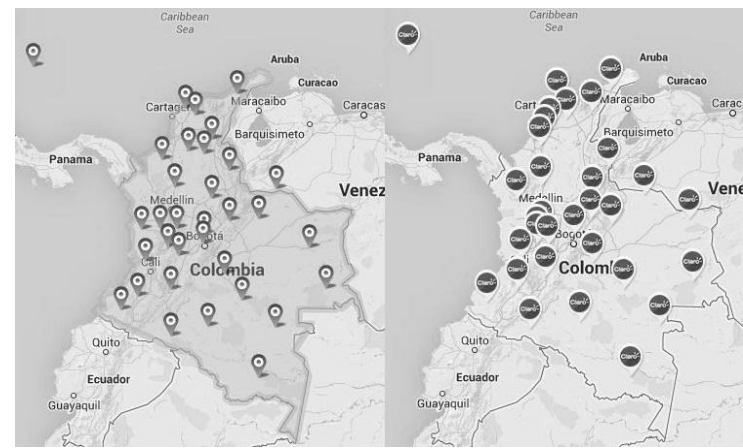

Fig. 2. (a)Cobertura Movistar (b) Cobertura Claro. Fuente: Movistar [10] Claro Colombia [11].

Una aproximación actual del área cubierta en el país por los distintos proveedores de servicios de conexión de datos por red celular se muestra en la Figura 1, esta información es tomada de la comunidad con teléfonos inteligentes con aplicaciones que reporten a la base de datos OpenSignal[8]. La Figura 2 es la información de cobertura otorgada por los proveedores del servicio para tecnologías $2 \mathrm{G}$ y superiores, aptas para el uso del sistema prototipado.

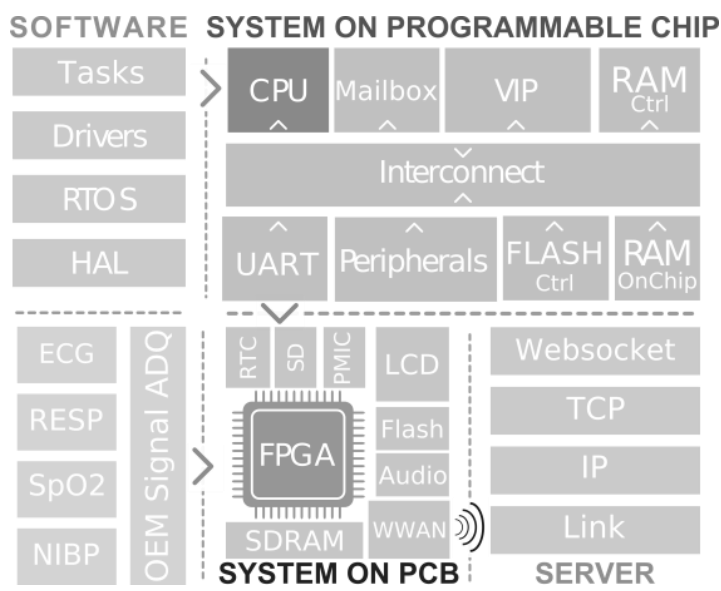

Figura 3. Diagrama del sistema MSV y Servidor. Fuente: Autor.
Para implementar la transmisión por red celular WWAN (Wireless Wide Area Network) de los datos adquiridos, se eligió un módulo que cumple con los requerimientos de capa física y arquitectura GSM y WCDMA. Este módulo se comunica con el procesador Nios II por protocolo UART (Universal Asynchronous Receiver/Transmitter) de 115200 Baudios y hace parte del equipo electrónico y sistema de telesalud del diagrama de la Figura 3.

Para la comunicación con el dispositivo se hace uso de comandos AT [9]. Estos permiten la configuración de la conexión en cuanto a APN (Access Point Name), tipo de antena utilizada, modo de escritura de datos (Transparente o por comandos), especificación de dirección IP (Internet Protocol) y puerto del servidor, conexión TCP (Transmission Control Protocol) y envío de datos correspondientes a los signos vitales leídos.

Estos comandos se agrupan para formar etapas genéricas para cualquier capa física, como se muestra en el diagrama de la figura 4. De esta forma el Software puede inicializar el Hardware de conexión (Encender el módulo y configurar la comunicación UART), Obtener IP por DHCP (Dynamic Host Configuration Protocol), iniciar conexión Websocket (Realizar conexión TCP y protocolo Handshake de Websocket), iniciar sesión en el servidor (enviar y confirmar mensajes de Login y Autenticación Websocket)[12] y enviar datos al mismo tiempo que verifica la comunicación con el servidor.

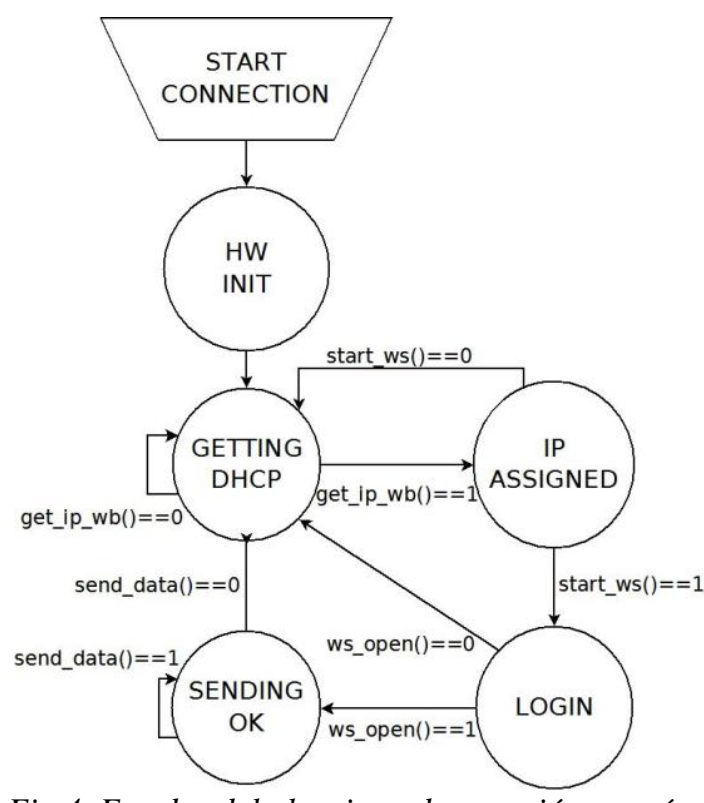

Fig.4. Estados del algoritmo de conexión y envío de datos.

Fuente: Autor. 


\subsection{Adquisición de signos vitales}

Los datos de parámetros fisiológicos son adquiridos de un módulo de OEM (Original Equipment Manufacturer) multi-parámetros que entrega por UART los datos procesados de un pulsioxímetro (SPO2), derivas de Electrocardiogra-fía (ECG), sensor de temperatura (TEMP) y brazalete de presión no invasiva (PANI). Como resultado se obtienen los datos estáticos de BPM (Beats Per Minute) por ECG y pulsioximetría, Saturación de Oxígeno (SpO2), frecuencia respiratoria (RR), temperatura en grados Celsius, presión Sistólica (SIS), Media (MAP) y Diastólica (DIAS) ; al igual que las ondas de 2 derivas ECG, $\mathrm{SpO} 2$ y Respiración. Estos paramatros fisiologicos son muy comunes en equipos comerciales de monitoreo de signos vitales, asi como tambien estan asociados a el estado general de un paciente[13], como se muestra en la Tabla 1.

Tabla 1.

Fisiología y factores de parametros fisiologicos Fuente: Critical care: the eight vital signs of patient monitoring.

\begin{tabular}{|c|c|c|}
\hline $\begin{array}{c}\text { Parámetro } \\
\text { fisiológico }\end{array}$ & Fisiología & Factores \\
\hline TEMP & $\begin{array}{c}\text { Controlada por el } \\
\text { hipotálamo }\end{array}$ & $\begin{array}{c}\text { Edad, Infección, } \\
\text { Medicamentos }\end{array}$ \\
\hline ECG & $\begin{array}{c}\text { Refleja el volumen } \\
\text { circulatorio y la fuerza de } \\
\text { contractibilidad }\end{array}$ & $\begin{array}{c}\text { Volumen intravascular, } \\
\text { Contractibilidad, } \\
\text { Demanda de oxigeno }\end{array}$ \\
\hline PANI & $\begin{array}{c}\text { Regulada por el centro } \\
\text { vasomotor en la medula }\end{array}$ & $\begin{array}{c}\text { Volumen intravascular, } \\
\text { Tono vascular, } \\
\text { Contractibilidad, }\end{array}$ \\
\hline RR & $\begin{array}{c}\text { Controlada por los centros } \\
\text { respiratorios en la medula } \\
\text { y el puente tronco- } \\
\text { encefálico (PONS) }\end{array}$ & $\begin{array}{c}\text { Hipercapnia, Hipoxemia, } \\
\text { Acidosis }\end{array}$ \\
\hline SPO2 & $\begin{array}{c}\text { Refleja la saturación de } \\
\text { oxigeno en la sangre }\end{array}$ & $\begin{array}{c}\text { Gasto cardiaco, Nivel de } \\
\text { hemoglobina, Fracción } \\
\text { de oxígeno inspirada }\end{array}$ \\
\hline
\end{tabular}

El módulo tiene una transmisión asíncrona de 115200 baudios utilizando un formato de encabezado o ID, datos, y suma de verificación o Checksum. Esta trama es decodificada por una Descripción de Hardware (HWD) en FPGA que reconoce cada ID y almacena los datos en On-Chip RAM (Random Access Memory) con conocimiento previo del tamaño e información de la trama; esta memoria es leída por el procesador tipo Soft-Core Nios II desde un segundo puerto a una razón distinta a la de escritura, por lo que la información de los parámetros fisiológicos está disponible en todo momento y puede ser leída durante el proceso de transmisión de datos sin que esto signifique un uso de recursos mayor que el de leer un puerto del procesador.
Los datos de ondas (2xECG/SpO2/RESP) se almacenan secuencialmente en un buffer doble de 500 datos por señal para permitir lectura y escritura simultánea. Estos 500 datos corresponden a 4 segundos de ondas.

\subsection{Funcionamiento en tiempo real}

El dispositivo electrónico utilizado en esta implementación cuenta, además de la adquisición y transmisión de datos, con una pantalla de visualización para datos estáticos y ondas, panel táctil, entradas de botones, reloj de tiempo real, sistema de archivos y manejo de potencia con batería. Es por esto que se hace necesario el uso de un RTOS para el manejo de distintas tareas, que en este caso es uC/OS II de la empresa Micrium.

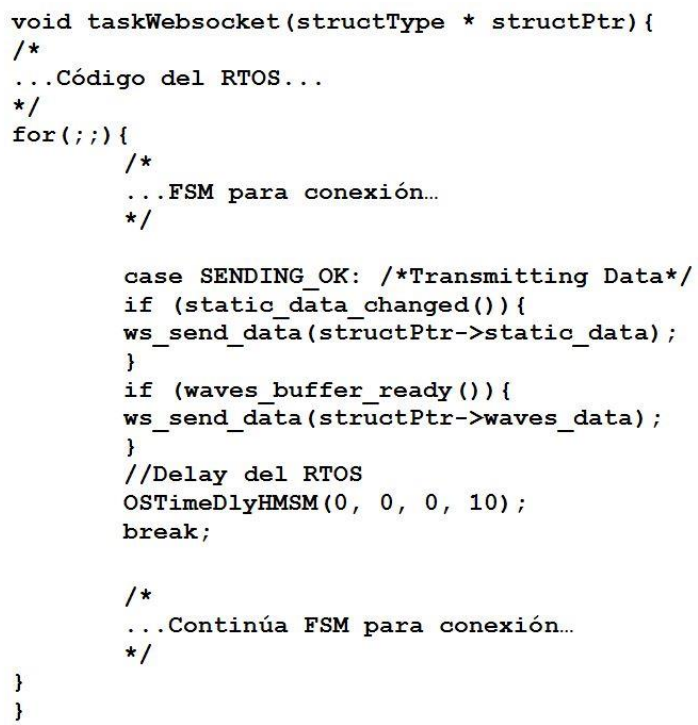

Fig. 5. Código RTOS.

Fuente: Autor.

Una de las tareas principales es la del control de transmisión de datos, lanzada desde la interfaz gráfica y encargada de realizar el encendido y configuración del módulo de conexión, creación y mantenimiento de la conexión TCP, y envío de tramas de Handshake, Login y paquetes de datos con empaquetamiento Websocket.

El código de la Figura 5 es un ejemplo de cómo se utilizan las funciones del RTOS para mantener la supervisión de las señales estáticas y ondas capturadas. Los datos estáticos se comparan con los valores anteriores antes de realizar su envío, de esta forma se mantiene un uso reducido de los recursos de red. Los datos de ondas se envían cuando el buffer entregue la señal de Ready, correspondiente a 500 datos de cada una de las ondas leídas. 


\subsection{Websocket}

La unidad base está implementada en un servidor $W e b$, esto permite el uso de la plataforma desde múltiples dispositivos a diferencia de una conexión punto a punto, la cual requiere de mayor capacidad de procesamiento por parte de la unidad de telesalud si se requiere la visualización de los datos por múltiples usuarios. Para su funcionamiento se hace uso del protocolo de comunicación Websocket con el fin de realizar una conexión persistente entre el cliente y servidor para el envío y recepción de información en Full-Duplex.

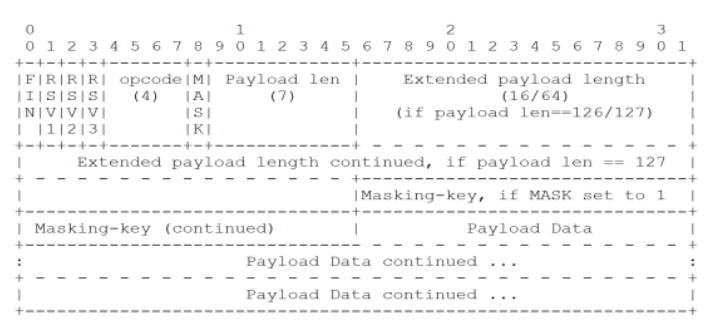

Fig. 6. Encabezado Websocket. Fuente: IETF-RFC 6455.

Websocket requiere que se cree una conexión TCP con el servidor y usando ese único puerto se realiza el envío de una trama HTTP (HyperText Transfer Protocol), llamada Handshake, que actualiza la conexión al protocolo Websocket. De forma similar existe una respuesta del servidor que confirma la actualización de la conexión. A partir de ese paso las tramas enviadas deben contar con el empaquetamiento que se muestra en la Figura 6 teniendo como carga útil o Payload la información en caracteres ASCII (American Standard Code for Information Interchange) de los parámetros fisiológicos simulados. Estos algoritmos fueron realizados en $\mathrm{C} / \mathrm{C}++$ para la unidad de telesalud con el procesador Nios II y se utiliza la herramienta jWebsocket para su procesamiento en el servidor o unidad base.

\section{RESULTADOS}

El prototipo desarrollado utiliza una tarjeta SIM (Subscriber Identification Module) del ISP (Internet Service Provider) Movistar de Colombia Telecomunicaciones, con un plan de servicios de navegación. El ISP presta los servicios de WCDMA y GSM sobre la frecuencia de $850 \mathrm{MHz}$.. Para el desarrollo de las pruebas se hace uso de la configuración en ambos tipos de conexión los cuales presentaron los resultados que se muestran en las Tablas 2 y 3.
La Tabla 2 muestra los tiempos de RTT (RoundTrip delay Time), resultado de la herramienta Ping utilizada desde la unidad de telesalud hacia al servidor de la unidad base. Esta prueba se realiza con GSM y WCDMA en condiciones de calidad de señal con RSSI (Received Signal Strength Indicator) entre $-55 \mathrm{dBm}$ y $-51 \mathrm{dBm}$.

Tabla 2. RTT por ping al servidor Fuente: Autor

\begin{tabular}{|c|c|c|c|}
\hline Red & RTTmin(ms) & RTTmed(ms) & RTTmáx(ms) \\
\hline GSM & 975 & 1189.6 & 1455 \\
\hline WCDMA & 101 & 113.125 & 200 \\
\hline
\end{tabular}

Una vez comprobada la comunicación por ambos tipos de conexión se realizó la integración con la unidad base. En esta, el programa jWebsocket reconoce únicamente tramas Websocket enviadas desde la unidad con el formato especificado previamente. La Figura 7 muestra el formato de la trama Handshake, con los campos variables entre símbolos "<” y “>”, para transmisión (NiosII $>$ ) y recepción (Module>).

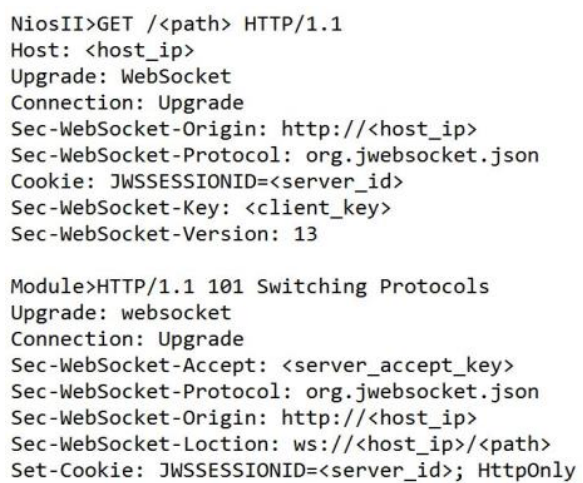

Fig. 7. Formato Handshake. Fuente: Autor.

Una vez realizada la conexión Websocket al servidor, la unidad de telesalud espera la solicitud desde la unidad base para realizar el envío de datos estáticos, límites y ondas. Los datos de ondas se activan al momento de recibir la primera solicitud de datos estáticos. Estas solicitudes se deben realizar con el encabezado de la Figura 6 y el Payload debe tener el formato que se muestra en la Figura 8. Los campos de los paquetes enviados por el monitor se muestran en la Tabla 4. Consisten de un encabezado que hace referencia a la implementacion de Websocket utilizada por la plataforma web (namespace), y un campo de tipo que define la distribucion de los siguientes elementos del paquete. Mantiene el uso de una cadena de caracteres, con los mismos tokens de inicio, final y separacion utilizados por el formato de solicitudes Websocket. 
Tabla 3. Latencia

\begin{tabular}{|c|c|}
\hline \multicolumn{2}{c}{ Fuente: Autor } \\
\hline Red & Latencia(ms) \\
\hline GSM & 1250 \\
\hline WCDMA & 130 \\
\hline
\end{tabular}

La Tabla 3 es resultado de la implementación de estos mensajes. El código en el envío de estas solicitudes inicia un contador en el momento anterior a la ejecución del mensaje y lo detiene cuando esa solicitud es recibida, obteniendo así la latencia del sistema de comunicación, lo cual incluye no sólo el retraso en la comunicación sino también los tiempos de ejecución por parte de la unidad de telesalud y la unidad base.

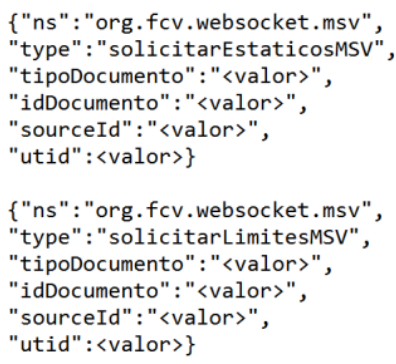

Fig. 8. Formato solicitudes Websocket Fuente: Autor.

\section{CONCLUSIONES}

Visualmente los resultados obtenidos con ambos tipos de conexión (GSM y CDMA) se mostraron diferentes, demostrando los valores obtenidos en la medición de latencia. Esto se puede observar en un retraso en la graficación de ondas al comparar la visualización web o de unidad base, como la que se muestra en la Figura 9, con la pantalla del equipo MSV durante la toma de datos de signos vitales, usando la conexión GSM. El retraso mayor a 1s es perceptible visualmente tanto en las gráficas de ondas como en los datos estáticos. La figura 10 compara el envío de una onda de ECG usando ambas redes y la respuesta en la interfaz web. Puede apreciarse como la transmisión por WCDMA toma menos tiempo para generar una respuesta en comparación con GSM.

La Figura 11 muestra un usuario utilizando el MSV con el sensor de pulsioximetría. Estos resultados permiten definir un uso de red fiable para cada tipo de conexión, en los cuales la red GSM puede ser utilizada para todos los valores estáticos, pero sólo 2 señales de onda en visualización, ya que la cantidad de datos de las 4 ondas simultaneas afectan el desempeño de la transmisión y recargan el buffer de salida del modulo.
Por otra parte, la conexión por WCDMA presenta un comportamiento óptimo para el envío de todas las señales obtenidas, valores estáticos y ondas ya que los retrasos no presentan un obstáculo en la comunicación, y la visualización de los mismos es estable.

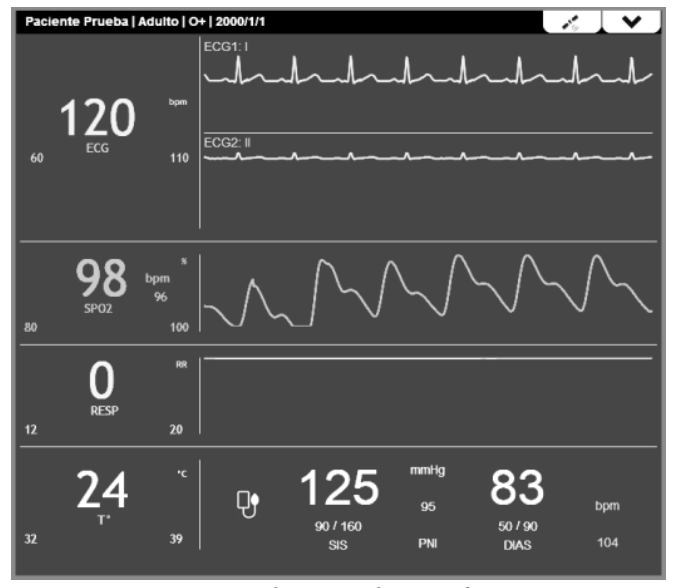

Fig. 9. Interfaz Web

Fuente: Autor.

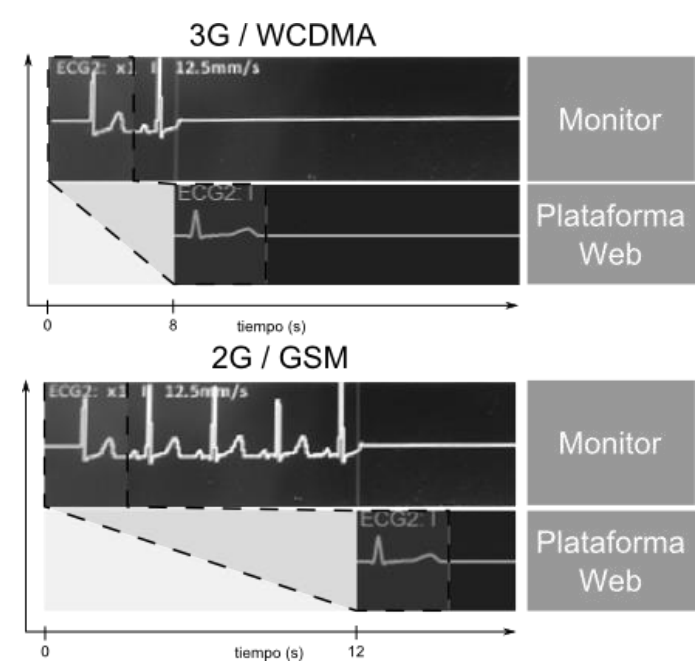

Fig. 10. Envío de datos por redes WCDMA y GSM. Fuente: Autor.

Este proyecto hace uso de tecnologías innovadoras que aún están emergiendo con gran impulso en aplicaciones de salud, como son las redes celulares y el desarrollo Web[14], además de proponer la implementación de FPGA como única unidad de procesamiento para la aceleración de tareas.

Esto permite una gran aplicabilidad en el entorno actual donde las soluciones de telesalud presentan una alternativa para ampliar y mejorar la atención médica en áreas remotas, donde no se cuenta con personal especializado, y aliviar la congestión de los centros médicos. 
Tabla 4. Campos paquetes Websocket Fuente: Autor.

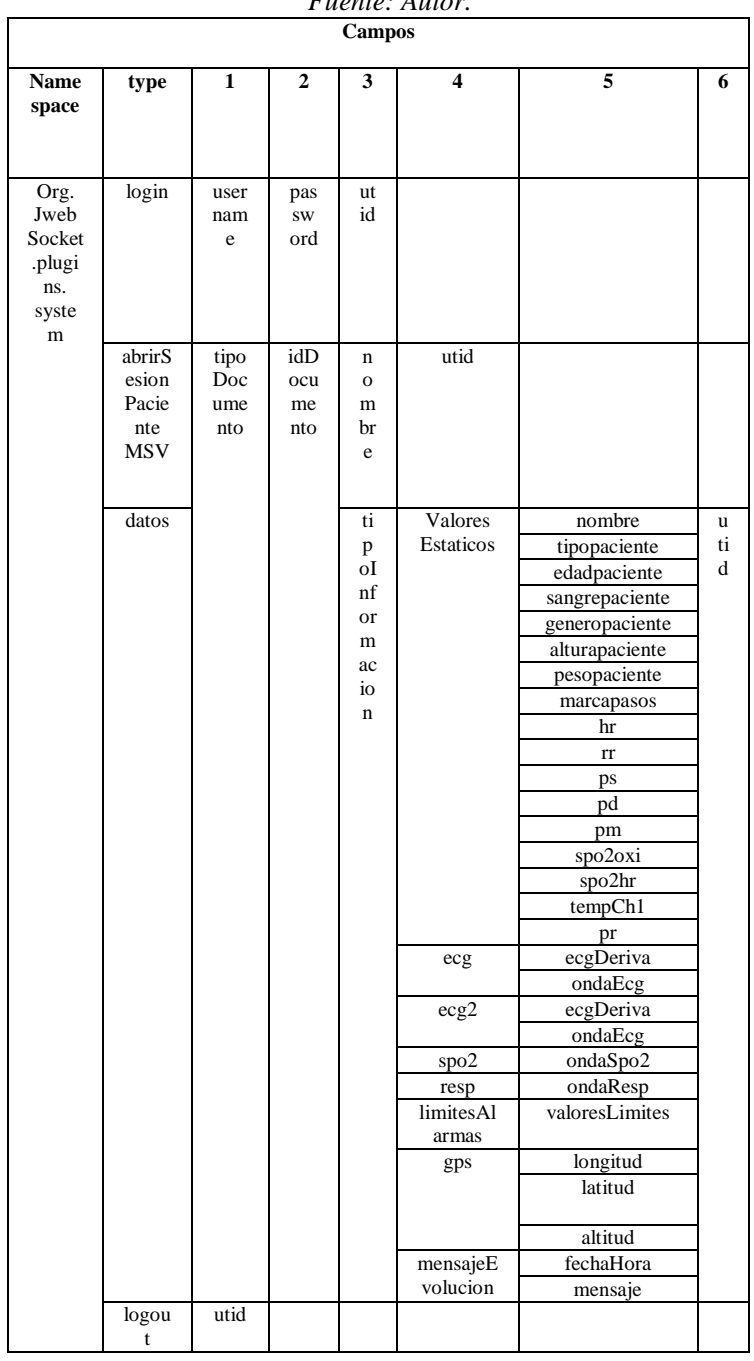

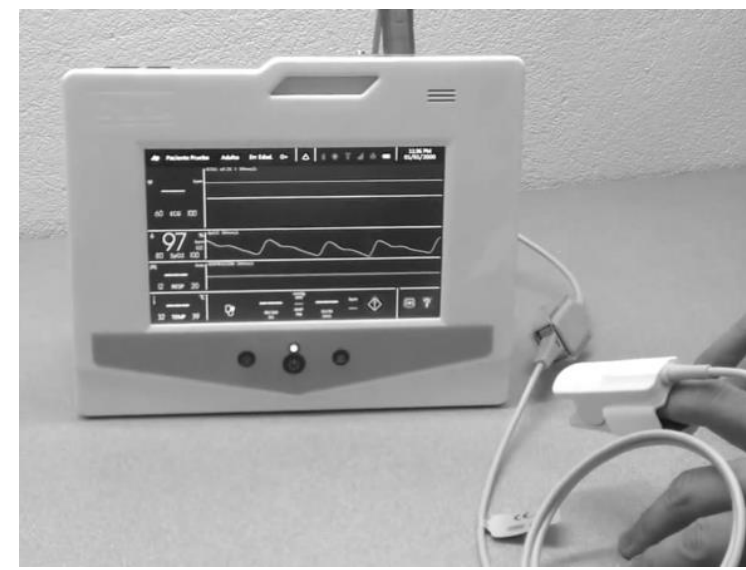

Fig. 11. Prototipo MSV. Fuente: Autor.

\section{AGRADECIMIENTOS}

Se agradece a Colciencias y al Programa de Jóvenes Investigadores e Innovadores, que en su convocatoria de 2012 favoreció la participación de Jose Pablo Pinilla en FCV Bioingeniería, bajo la tutoría de Oscar Mantilla en el desarrollo documentado en esta publicación.

\section{REFERENCIAS}

[1] B. Kumar, S.P. Singh, Mohan, A., "Emerging mobile communication technologies for health". En International Conference on Computer and Communication Technology (ICCCT 2010), Allahabad (India), 2010, pp. 828-832. 17-19.

[2] "Embarking on the Journey for Virtual Care 2012/13 Annual Report", OTN, Ontario, 2013.

[3] "SARURO Tele Care Box (TCB-081) Dispositivo de Telemonitorización de Signos Vitales", UNAL - Centro de Telemedicina, Bogotá (Colombia), 2008.

[4] M. C. Ardila, J. E. Ayala, C. M. Amaya, "Tele Unidad de Cuidado Intensivo, Resolviendo Paradigmas". F.C.C., 2011.

[5] "Nios II Processor Reference Handbook", Altera Corporation, NII5V1-13.1, USA, 2014.

[6] "SIM5218A Hardware Design", SIMCom, SIM5218A_HD_V1.06, 2010.

[7] "Vive Digital Colombia: Documento vivo del plan versión 1.0/ Febrero de 2011", Ministerio de Tecnologías de la información y las telecomunicaciones, Colombia, 2011.

[8] OpenSignal, $3 G$ and $4 G$ LTE Cell Coverage Map - OpenSignal, Fecha de Acceso: 20/03/2014, http://opensignal.com/

[9] Movistar Colombia, Cobertura - Movistar Colombia, Fecha de Acceso: 20/03/2014, http://www.movistar.co/internet/Internet_para _tu_celular/Cobertura/

[10] Claro Colombia, Mapa Cobertura Soluciones Móviles, Fecha de Acceso: 20/03/2014, http://www.claro.com.co/wps/portal/co/pc/pers onas/ayuda/cobertura-movil

[11] "SIM5218 AT Command Set", SIMCom, SIM5218_Serial_ATC_V1.38, 2012.

[12] I. Fette, A. Melkinov, "The Websocket Protocol", IETF, RFC 6455, 2011.

[13] Elliott M, Coventry A: "Critical care: the eight vital signs of patient monitoring". British Journal of Nursing 2012, 21(10), pp. 621-625.

[14] "Emerging Technology in Health Engagement", WorldatWork, USA, 2013. 